\title{
Ground State Structure of a Coupled 2-Fermion System in Supersymmetric Quantum Mechanics
}

\author{
Felix Finster \\ Harvard University, Department of Mathematics
}

November 1996

\begin{abstract}
We prove the uniqueness of the ground state for a supersymmetric quantum mechanical system of two fermions and two bosons, which is closely related to the $N=1 \mathrm{WZ}$-model. The proof is constructive and gives detailed information on what the ground state looks like.
\end{abstract}

\section{Introduction, definition of the model}

A supersymmetric quantum system is defined by two self-adjoint operators $Q, \gamma$ on a Hilbert space $\mathcal{H}$ satisfying the relations

$$
\gamma^{2}=1 \quad, \quad\{\gamma, Q\}=0
$$

They are called supercharge and grading operator. The Hamiltonian is given by $H:=Q^{2} \geq 0$. It is convenient to decompose $\mathcal{H}=\mathcal{H}^{+} \oplus \mathcal{H}^{-}$into the eigenspaces of $\gamma$, which leads to the $(2 \times 2)$-matrix representations

$$
\gamma=\left(\begin{array}{cc}
1 & 0 \\
0 & -1
\end{array}\right), \quad Q=\left(\begin{array}{cc}
0 & Q^{-} \\
Q^{+} & 0
\end{array}\right), \quad H=\left(\begin{array}{cc}
Q^{-} Q^{+} & 0 \\
0 & Q^{+} Q^{-}
\end{array}\right)
$$

Eigenstates of $H$ with zero eigenvalue are called ground states. The ground state structure of supersymmetric systems has been studied intensively (see e.g. [1], [2], [5], [6], [7]). It is of particular interest because it tells about a spontaneous breaking of the supersymmetry (see e.g. [3]). In this paper we will analyze the system where $Q^{ \pm}$are the differential operators on $L^{2}\left(\mathbb{R}^{2}\right)^{2}$

$$
\begin{aligned}
Q^{+} & =-i\left(\begin{array}{cc}
\partial_{z} & 0 \\
0 & \partial_{\bar{z}}
\end{array}\right)-i\left(\begin{array}{cc}
0 & \partial_{z} W \\
\partial_{\bar{z}} W & 0
\end{array}\right) \\
Q^{-} & =\left(Q^{+}\right)^{*}=-i\left(\begin{array}{cc}
\partial_{\bar{z}} & 0 \\
0 & \partial_{z}
\end{array}\right)+i\left(\begin{array}{cc}
0 & \partial_{z} W \\
\partial_{\bar{z}} \bar{W} & 0
\end{array}\right)
\end{aligned}
$$

(we use the complex notation $z=x+i y, \bar{z}=x-i y, \partial_{z}=\frac{1}{2}\left(\partial_{x}-i \partial_{y}\right), \partial_{\bar{z}}=\frac{1}{2}\left(\partial_{x}+i \partial_{y}\right)$ ). The superpotential $W=W(|z|)$ is assumed to be a complex polynomial of degree at least two. 
As motivation we explain, in which context this system arises and why it is of some general interest: One of the simplest examples of a supersymmetric quantum field theory is the $N=1$ WZ-model on the cylinder. It is defined by

$$
Q=\int_{S^{1}} d x\left(\Psi_{1}(x) \pi(x)-\Psi_{2}(x) \partial_{x} \varphi(x)+\Psi_{2}(x) V^{\prime}(\varphi(x))\right)
$$

where the field operators $\Psi_{j}, \pi, \varphi$ satisfy the canonical (anti-)commutation relations

$$
\begin{aligned}
\left\{\Psi_{i}(x), \Psi_{j}(y)\right\} & =\delta_{i j} \delta(x, y), \quad[\pi(x), \varphi(y)]=-i \delta(x, y) \\
{\left[\Psi_{i}(x), \pi(y)\right] } & =\left[\Psi_{i}(x), \varphi(y)\right]=0
\end{aligned}
$$

The analysis of the ground state structure of this system turns out to be an involved problem. If the potential $V$ is sufficiently small, cluster expansion methods can be used to prove the uniqueness of the ground state in the infinite volume limit (see [8]). The general difficulty is the complicated coupling of the quantum fields. To make this more apparent, we expand the field operators in a Fourier series

$$
f(x)=\frac{1}{\sqrt{2 \pi}} \sum_{k \in \mathbf{Z}} f(k) e^{-i k x},
$$

which leads to the equation

$$
Q=\sum_{k \in \mathbf{Z}}\left(\Psi_{1}(k) \pi(-k)-i k \Psi_{2}(k) \varphi(-k)+\Psi_{2}(k) V^{\prime}(-k)\right)
$$

The Fourier modes are mixed up in the sum and cannot be easily separated. We can view the operators $\Psi_{1}(k), \Psi_{2}(-k)$ as the annihilation/creation-operators of a fermion with momentum $k$, conversely $\pi(k), \varphi(-k)$ are the canonical variables of a $k$-momentum boson. With this picture, (3) describes a strongly interacting fermion/boson-system.

A rough simplification of the problem consists in the reduction to the zero modes,

$$
Q=\Psi_{1}(0) \pi(0)+\Psi_{2}(0) V^{\prime}(0) \quad .
$$

This is $N=1$ quantum mechanics 沟, as one sees after rewriting $\varphi, \pi$ as canonical multiplication/differential operators on $L^{2}(\mathbb{R})$ and choosing a matrix representation for the fermionic operators $\Psi_{j}$. Unfortunately the coupling structure of the different Fourier modes in (3) gets completely lost in this limit. Therefore it seems interesting to study the system of two coupled Fourier modes,

$$
Q=\sum_{k= \pm 1}\left(\Psi_{1}(k) \pi(-k)-i k \Psi_{2}(k) \varphi(-k)+\Psi_{2}(k) V^{\prime}(-k)\right)
$$

This model shows many characteristics of the field theory (3), but is still simple enough for a detailed analysis. In a suitable representation of the field operators, it coincides with the system (11), (2) if we choose $W(r)=r^{2}+i V(r)$.

We conclude that the differential operators (1), (2) describe a supersymmetric quantum mechanical system of two fermions and two bosons. The coupling is typical for supersymmetric field theories and should be helpful for the understanding of these systems. 


\section{Reduction to a one-dimensional problem}

We start the analysis of the ground states with some explicit calculations. Since the equations $H \Psi=0, Q \Psi=0$ are equivalent, it suffices to look for solutions of the equations

$$
Q^{ \pm} \Psi^{ \pm}=0 \quad \text { with } \quad \Psi^{ \pm} \in L^{2}\left(\mathbb{R}^{2}\right)^{2}
$$

Because of the radial symmetry of $W$, it is useful to choose polar coordinates $(r, \varphi)$. We have

$$
\partial_{z}=\frac{e^{-i \varphi}}{2}\left(\partial_{r}-\frac{i}{r} \partial_{\varphi}\right), \quad \partial_{\bar{z}}=\frac{e^{i \varphi}}{2}\left(\partial_{r}+\frac{i}{r} \partial_{\varphi}\right)
$$

and thus

$$
\begin{aligned}
Q^{+} & =-\frac{i}{2}\left(\begin{array}{cc}
e^{-i \varphi} \partial_{r} & e^{-i \varphi} W^{\prime} \\
e^{i \varphi} \overline{W^{\prime}} & e^{i \varphi} \partial_{r}
\end{array}\right)+\frac{1}{2 r}\left(\begin{array}{cc}
-e^{-i \varphi} & 0 \\
0 & e^{i \varphi}
\end{array}\right) \partial_{\varphi} \\
Q^{-} & =-\frac{i}{2}\left(\begin{array}{cc}
e^{i \varphi} \partial_{r} & -e^{-i \varphi} W^{\prime} \\
-e^{i \varphi} \bar{W}^{\prime} & e^{-i \varphi} \partial_{r}
\end{array}\right)+\frac{1}{2 r}\left(\begin{array}{cc}
e^{i \varphi} & 0 \\
0 & -e^{-i \varphi}
\end{array}\right) \partial_{\varphi}
\end{aligned}
$$

We multiply the equation $Q^{+} \Psi^{+}=0$ with the matrix $2 i \operatorname{diag}\left(e^{i \varphi}, e^{-i \varphi}\right)$,

$$
\left[\partial_{r}-\frac{i}{r}\left(\begin{array}{cc}
1 & 0 \\
0 & -1
\end{array}\right) \partial_{\varphi}+\left(\begin{array}{cc}
0 & W^{\prime} \\
W^{\prime} & 0
\end{array}\right)\right] \Psi^{+}=0
$$

The differential operator in the square bracket commutes with angular momentum $i \partial_{\varphi}$. Thus we can make the separation ansatz

$$
\Psi^{+}(r, \varphi)=\frac{1}{\sqrt{2 \pi}} \sum_{m \in \mathbf{Z}} \Psi_{m}^{+}(r) e^{-i m \varphi}
$$

and obtain the ordinary differential equations $A_{m} \Psi_{m}^{+}=0$ with

$$
A_{m}(r)=\frac{d}{d r}-\frac{m}{r}\left(\begin{array}{cc}
1 & 0 \\
0 & -1
\end{array}\right)+\left(\begin{array}{cc}
0 & W^{\prime} \\
W^{\prime} & 0
\end{array}\right)
$$

For the equation $Q^{-} \Psi^{-}=0$ we substitute

$$
\Psi^{-}(r, \varphi)=\frac{1}{r}\left(\begin{array}{cc}
e^{-i \varphi} & 0 \\
0 & -e^{i \varphi}
\end{array}\right) \Phi^{-}(r, \varphi) \quad .
$$

The resulting equation for $\Phi^{-}$has, after multiplication with the matrix $2 \operatorname{ir} \operatorname{diag}(1,-1)$, the form

$$
\left[\partial_{r}+\frac{i}{r}\left(\begin{array}{cc}
1 & 0 \\
0 & -1
\end{array}\right) \partial_{\varphi}+\left(\begin{array}{cc}
0 & W^{\prime} \\
\bar{W}^{\prime} & 0
\end{array}\right)\right] \Phi^{-}=0
$$

After separating the variables by

$$
\Phi^{-}(r, \varphi)=\frac{1}{\sqrt{2 \pi}} \sum_{m \in \mathbf{Z}} \Phi_{m}^{-}(r) e^{i m \varphi},
$$


the radial functions $\Phi_{m}^{-}$must satisfy the equations $A_{m} \Phi_{m}^{-}=0$.

In this way we have reduced the problem to the analysis of the ordinary differential equations

$$
A_{m} f_{m}=0 \quad, \quad f \in C^{1}\left(\mathbb{R}^{+}\right)^{2} \quad .
$$

The conditions $\Psi^{ \pm} \in L^{2}\left(\mathbb{R}^{2}\right)^{2}$ mean, according to (4), (6), (7), that the solutions $\Psi_{m}^{+}, \Phi_{m}^{-}$must satisfy the conditions

$$
\begin{aligned}
& \int_{0}^{\infty}\left|\Psi_{m}^{+}\right|^{2} r d r \leq \infty \\
& \int_{0}^{\infty}\left|\Phi_{m}^{-}\right|^{2} \frac{d r}{r} \leq \infty
\end{aligned}
$$

It remains to find out for which values $m \in \mathbf{Z}$ there are solutions of (8) satisfying (9) resp. (10).

\section{A differential inequality independent of $W$}

We define for a given solution $f_{m}$ of (8) the real function $\Lambda_{f_{m}}$ by

$$
\Lambda_{f_{m}}(r)=<f_{m}(r),\left(\begin{array}{cc}
1 & 0 \\
0 & -1
\end{array}\right) f_{m}(r)>\quad .
$$

It satisfies the equation

$$
\begin{aligned}
\frac{d}{d r} \Lambda_{f_{m}}= & \frac{2 m}{r}<f_{m}, f_{m}> \\
& -<\left(\begin{array}{cc}
0 & W^{\prime} \\
\bar{W}^{\prime} & 0
\end{array}\right) f_{m},\left(\begin{array}{cc}
1 & 0 \\
0 & -1
\end{array}\right) f_{m}>-<f_{m},\left(\begin{array}{cc}
1 & 0 \\
0 & -1
\end{array}\right)\left(\begin{array}{cc}
0 & W^{\prime} \\
W^{\prime} & 0
\end{array}\right) f_{m}> \\
= & \frac{2 m}{r}<f_{m}, f_{m}>
\end{aligned}
$$

and thus

$$
\frac{d}{d r} \Lambda_{f_{m}} \geq \frac{2 m}{r}\left|\Lambda_{f_{m}}\right|
$$

This inequality implies that $m$ vanishes for all ground states:

Lemma 3.1 For $m \neq 0$ all (nontrivial) solutions of (\$) violate both (\$) and (10).

Proof: Suppose that $m \neq 0$ and that a solution $f_{m}$ of (8) satisfies either (9) or (10). We can assume that $m>0$, because we can otherwise replace $\Lambda_{f_{m}}$ by $-\Lambda_{f_{m}}$ and multiply (11) by a factor -1 . According to (12), the function $\Lambda_{f_{m}}$ is monotonely increasing.

Suppose that there is $r_{0}>0$ with $\Lambda_{f_{m}}\left(r_{0}\right)>0$. Then $\Lambda_{f_{m}}(r)>0$ for all $r>r_{0}$ and

$$
\frac{d}{d r} \Lambda_{f_{m}}(r) \geq \frac{2 m}{r} \Lambda_{f_{m}}(r)
$$

Integrating this inequality yields the bound

$$
\Lambda_{f_{m}}(r) \geq \Lambda_{f_{m}}\left(r_{0}\right)\left(\frac{r}{r_{0}}\right)^{2 m} \quad \text { for } r \geq r_{0}
$$


Since $\left|f_{m}(r)\right|^{2} \geq \Lambda_{f_{m}}(r)$, the function $\left|f_{m}(r)\right|^{2}$ grows at least quadratic at infinity, which contradicts (9), (10).

Suppose conversely that there is $r_{0}>0$ with $\Lambda_{f_{m}}\left(r_{0}\right)<0$. Then $\Lambda_{f_{m}}(r)<0$ for all $r<r_{0}$ and

$$
\frac{d}{d r} \Lambda_{f_{m}}(r) \geq-\frac{2 m}{r} \Lambda_{f_{m}}(r)
$$

We thus have the bound

$$
\Lambda_{f_{m}}(r) \leq \Lambda_{f_{m}}\left(r_{0}\right)\left(\frac{r_{0}}{r}\right)^{2 m}
$$

which imples that $\left|f_{m}(r)\right|^{2}$ has at least a quadratic pole at the origin. This is again a contradiction to (9), (10).

We conclude that $\Lambda_{f_{m}}$ must vanish identically. But then, according to (11), $f_{m} \equiv 0$.

According to this Lemma it remains to analyze the equation $A_{0} f=0$. Since the operator $A_{0}$ is regular for $r=0$, the solutions $f$ have no singularities at the origin, $f \in C^{1}\left(\mathbb{R}_{0}^{+}\right)$.

\section{The equation $Q^{-} \Psi^{-}=0$ only has the trivial solution}

Let $\Phi_{0}^{-}$be a solution of the equation $A_{0} \Phi_{0}^{-}=0$ satisfying (10). Because of the divergent factor $r^{-1}$ in (10),$\Phi^{-}$must vanish at the origin. Hence, by the uniqueness theorem for ordinary differential equations, $\Phi_{0}^{-} \equiv 0$.

\section{The equation $Q^{+} \Psi^{+}=0$ has a unique solution}

Let $\Psi_{0}^{+}$be a solution of the equation $A_{0} \Psi_{0}^{+}=0$ satisfying (9).

We use the scalar equation (11) to reduce the degrees of freedom of $\Psi_{0}^{+}$: For $m=0$, (11) implies that $\Lambda_{\Psi_{0}^{+}}(r)$ is a constant. Since $\left|\Psi_{0}^{+}\right|^{2} \mid \geq \Lambda_{\Psi_{0}^{+}}$, (9) can only be satisfied if this constant is zero, $\Lambda_{\Psi_{0}^{+}} \equiv 0$. Hence we can represent $\Psi_{0}^{+}$in the form

$$
\Psi_{0}^{+}(r)=\left(\begin{array}{c}
u(r) \\
u(r) e^{-i \alpha(r)}
\end{array}\right)
$$

with a complex function $u(r)$ and a real function $\alpha(r)$. Furthermore we choose a polar representation

$$
W^{\prime}=U(r) e^{i \beta(r)}
$$

of $W^{\prime}$ with smooth real functions $U, \beta$. We can assume that $U(r)$ is positive for large $r$. After these transformations, the equation $A_{0} \Psi_{0}^{+}$takes the form

$$
\begin{aligned}
\frac{d}{d r} \ln (u) & =-U e^{-i(\alpha-\beta)} \\
\frac{d}{d r} \ln (u)-i \alpha^{\prime} & =-U e^{i(\alpha-\beta)}
\end{aligned}
$$


It will turn out to be more convenient to choose $\gamma:=\alpha-\beta$ as the variable describing the relative phase in (13). Then we get the system of differential operators

$$
\begin{aligned}
\gamma^{\prime} & =2 U \sin (\gamma)-\beta^{\prime} \\
\frac{d}{d r} \ln u & =-U e^{-i \gamma} .
\end{aligned}
$$

The condition (9) transforms into

$$
\int_{0}^{\infty}|u|^{2} r d r<\infty
$$

This construction has simplified the equations considerably. Since (14) does not depend on $u$, we can study the equation for $\gamma$ independently. For given $\gamma$, we can solve (15) by integration,

$$
u(r)=c \exp \left(-\int_{0}^{r} U(\tau) e^{-i \gamma(\tau)} d \tau\right) \quad, \quad c \in \mathbb{C} .
$$

The condition (16) can be reformulated with the asymptotic behaviour of $\gamma$ : Since (14), (15) are regular at the origin, (16) only yields a condition on the decay of $u$ at infinity. Suppose that $\gamma(r) \in\left(-\frac{\pi}{2}, \frac{\pi}{2}\right)(\bmod 2 \pi)$ for large $r$. Then (17) decays exponentially at infinity, and (16) will be satisfied. If, conversely, $\gamma(r) \in\left(\frac{\pi}{2}, \frac{3 \pi}{2}\right)(\bmod 2 \pi)$ for large $r$, the function $u(r)$ will grow exponentially, and (16) will be violated.

We have thus reduced the problem to the analysis of the nonlinear scalar equation (14). It is useful to view this equation as a dynamical system. In the special case $\beta^{\prime} \equiv 0$, the equation $\gamma^{\prime}=2 U \sin (\gamma)$ has the two fixed points $\gamma=0, \pi(\bmod 2 \pi)$, which are repulsive and attractive respectively. Every solution $\gamma$ with $\gamma(r) \neq 0$ will run into the attractive fixed point, $\lim _{r \rightarrow \infty} \gamma(r)=\pi$. We conclude that (16) implies that $\gamma \equiv 0(\bmod 2 \pi)$. The equation for $u$ then has (up to normalization) the unique solution

$$
u(r)=\exp \left(-\int_{0}^{r} U(\tau) d \tau\right)
$$

and we thus have exactly one ground state.

In the general case, equation (14) is more complicated. Fortunately we can apply a perturbation argument: In the limit $r \rightarrow \infty, \beta(r)$ converges to a phase which is determined by the coefficient of the highest power of $W$. As consequence, $\beta^{\prime}$ decays at least quadratic at infinity, i.e.

$$
\left|\beta^{\prime}(r)\right| \leq \frac{C}{r^{2}}
$$

for a suitable constant $C$. Since we must only consider the situation for large $r$, we can view the summand $-\beta^{\prime}$ in (14) as being arbitrarily small. Therefore we can still expect a unique solution $\gamma$ with $\lim _{r \rightarrow \infty} \gamma(r)=0(\bmod 2 \pi)$. This consideration is made mathematically precise in the following lemma.

Lemma 5.1 The equation (14) has a unique solution $\gamma$ with $\lim _{r \rightarrow \infty} \gamma(r)=0(\bmod 2 \pi)$. For all other solutions $\bar{\gamma}, \lim _{r \rightarrow \infty} \bar{\gamma}(r)=\pi(\bmod 2 \pi)$. 
Proof: We define the (nonlinear) operator $N$ by

$$
N: H^{1,1}\left(\mathbb{R}^{+}\right) \rightarrow L^{1}\left(\mathbb{R}^{+}\right): f \rightarrow f^{\prime}-\sin (f) \quad .
$$

$N$ is differentiable at the origin and $d N_{\mid 0} f=f^{\prime}-f$. For $g \in L^{1}\left(\mathbb{R}^{+}\right)$let $f$ be the function

$$
f(y)=-\int_{y}^{\infty} d x e^{y-x} g(x)
$$

The calculation

$$
\begin{aligned}
\int_{0}^{\infty}|f(y)| d y & \leq \int_{0}^{\infty} d y \int_{y}^{\infty} d x e^{y-x}|g(x)|=\int_{0}^{\infty} d x \int_{0}^{x} d y e^{y-x}|g(x)| \\
& =\int_{0}^{\infty} d x\left(e^{x}-1\right) e^{-x}|g(x)| \leq 2\|g\|_{1}
\end{aligned}
$$

shows that $f \in H^{1,1}(\mathbb{R})$. Furthermore $d N_{\mid 0} f=g$, and thus $d N_{\mid 0}$ is a bijection. As consequence there are neighborhoods $U \subset H^{1,1}\left(\mathbb{R}^{+}\right), V \subset L^{1}\left(\mathbb{R}^{+}\right)$of the origin such that $N: U \rightarrow V$ is a homeomorphism.

We now construct $\gamma$ : For $r_{0}$ large enough, $U(r)$ is positive and monotone for all $r>r_{0}$. We introduce a new variable $s$ by

$$
s(r)=U^{-1}\left(\frac{r}{2}\right) \quad, \quad s_{0}:=s\left(r_{0}\right)
$$

and rescale (14),

$$
\gamma^{\prime}(s)=\sin (\gamma)+g(s) \quad \text { with } \quad g(s)=-\frac{\beta^{\prime}}{2 U} \quad, \quad s \geq s_{0} \quad .
$$

The function $g$ is in $L^{1}\left(\left(s_{0}, \infty\right)\right)$. By further increasing $s_{0}$, we can arrange that $g\left(\cdot-s_{0}\right) \in V$. Then $\gamma(r):=N^{-1}(g)\left(r+r_{0}\right) \in H^{1,1}\left(\left(r_{0}, \infty\right)\right)$ satisfies (14) for $r \geq r_{0}$. Finally we extend this solution to $H^{1,1}\left(\mathbb{R}^{+}\right)$.

To prove uniqueness let $\bar{\gamma}$ be a solution of (14) with $\lim _{r \rightarrow \infty} \neq \pi(\bmod 2 \pi)$. Then $\bar{\gamma}(r) \in$ $\left(-2 C / r^{2}, 2 C / r^{2}\right)(\bmod 2 \pi)$, because otherwise $\bar{\gamma}$ will run into the attractive fixed point. Thus $\bar{\gamma}\left(\cdot+r_{0}\right) \in V$ for $r_{0}$ large enough, and the injectivity of $N_{\mid V}$ implies that $\bar{\gamma}=\gamma$.

We conclude that the equations (14), (15) have (up to normalization) a unique solution satisfying (16). We substitute (17), (13) into (4) and obtain for the ground state the formula

$$
\Psi^{+}(r, \varphi)=c \exp \left(-\int_{0}^{r} U(\tau) e^{-i \gamma(\tau)} d \tau\right)\left(\begin{array}{c}
1 \\
e^{-i(\beta+\gamma)}
\end{array}\right)
$$

\section{Discussion}

We come to a brief discussion of the ground state. According to (19), $\Psi^{+}$is spherically symmetric, decays exponentially at infinity and is regular at the origin. We first look qualitatively at regions where the eigenvectors of the superpotential do not depend much on $x$ (the eigenvalues 
can be arbitrary). In this case $\beta^{\prime}$ is small and consequently, $\gamma \approx 0$ (to see this, consider (14) backwards in $r$ ). We thus have the formula

$$
\Psi^{+}(r, \varphi) \approx c \exp \left(-\int^{r}|W|\right)\left(\begin{array}{c}
1 \\
e^{-i \beta}
\end{array}\right) \quad .
$$

$\Psi^{+}(r, \varphi)$ is proportional to the lowest eigenvector of the superpotential, the factor $\exp \left(-\int|W|\right)$ is similar to the ground state of $N=1$ quantum mechanics. In regions where the eigenvectors of the superpotential are strongly fluctuating, however, $\gamma$ can become large. In this case, the spinorial dependence of $\Psi^{+}$no longer minimizes the expectation value of the superpotential. The wave-function feels an "effective" potential described by the additional phase factor $e^{-i \gamma}$ in (19). Nevertheless, the ground state energy remains zero.

We see that, in contrast to $N=1$ quantum mechanics, the existence of a ground state does not depend on the degree of the superpotential. On the other hand, our result is a confirmation for the hypothesis expressed in [2] that the $N=1 \mathrm{WZ}$-model on the cylinder has only one ground state, even if the potential has several minima.

\section{References}

[1] M. Claudson, M. Halpern: Supersymmetric ground state wave functions. Nucl. Phys. B250, 689-715 (1985)

[2] S. Janowky, J. Weitsman: The phase structure of the two-dimensional WZ-model on a cylinder. Harvard University Preprint 1990

[3] A. Jaffe, A. Lesniewski: Supersymmetric quantum fields and infinite-dimensional analysis, 1987 Cargese Lecture Notes, Preprint HUTMP B-210

[4] A. Jaffe, A. Lesniewski, M. Lewenstein: Ground state structure in supersymmetric quantum mechanics, Ann. Phys. 178, 313-329 (1987)

[5] A. Jaffe, A. Lesniewski, J. Weitsman: Index of a family of Dirac operators on loop space, Commun. Math. Phys. 112, 75-88 (1987)

[6] A. Jaffe, A. Lesniewski, J. Weitsman: The two-dimensional, $N=2$ WZ-model on a cylinder, Commun. Math. Phys. 114, 147-166 (1988)

[7] A. Jaffe, A. Lesniewski, J. Weitsman: The $S^{1} \rightarrow \mathbb{R}$ loop space and supersymmetric quantum fields, Ann. Phys. 183, 337-351 (1988)

[8] J. Weitsman: A supersymmetric field theory in infinite volume. Harvard University Thesis, 1990 